\title{
Unsteady Jeffrey-Hamel Flow in the Presence of Oblique Magnetic Field with Suction and Injection
}

\author{
Edward Richard Onyango ${ }^{1,}$, , Mathew Ngugi Kinyanjui ${ }^{1}$, Mark Kimathi ${ }^{1,2}$, \\ Surindar Mohan Uppal ${ }^{1}$ \\ ${ }^{1}$ Department of Pure and Applied Mathematics, Jomo Kenyatta University of Agriculture and Technology, Nairobi, Kenya \\ ${ }^{2}$ Department of Mathematics, Statistics and Actuarial Science, Machakos University, Machakos, Kenya
}

Email address:

edwardrichard36@gmail.com (E. R. Onyango)

${ }^{*}$ Corresponding author

\section{To cite this article:}

Edward Richard Onyango, Mathew Ngugi Kinyanjui, Mark Kimathi, Surindar Mohan Uppal. Unsteady Jeffrey-Hamel Flow in the Presence of Oblique Magnetic Field with Suction and Injection. Applied and Computational Mathematics. Vol. 9, No. 1, 2020, pp. 1-13. doi: 10.11648/j.acm.20200901.11

Received: February 3, 2020; Accepted: February 13, 2020; Published: February 25, 2020

\begin{abstract}
In this study, the magnetohydrodynamic flow of an incompressible, viscous electrically conducting fluid through a convergent-divergent channel in the presence of an oblique variable magnetic field to the flow with a case of suction and injection on the walls has been investigated. The velocity profiles, temperature profiles, the effects of injection and suction, time, induced magnetic field and the effects of varying various parameters on the flow have been investigated. The equations governing the MHD flow are solved by the collocation method and the results presented in graphs. The velocity, temperature, and magnetic induction increases with the increase in the suction parameter and decrease in the wedge angle while velocity, temperature, and magnetic induction reduce with the increase in the injection parameter. The velocity, temperature and magnetic induction increase with the increase in the Hartmann number. The results of this study will be useful information to the engineers to improve the performance and efficiency of machines in the industrial, environmental, aerospace, chemical, civil, mechanical and biomechanical engineering applications.
\end{abstract}

Keywords: Jeffrey-Hamel Flow, Newtonian Fluid, Inclined Magnetic Field, Unsteadiness Parameter

\section{Introduction}

The problem of investigating incompressible Newtonian fluid flows and their characteristics between nonparallel walls has extensively generated much importance in recent years. In the recent past years, considerable efforts have been put to study the various models related to the Newtonian fluids and to consider the flow behavior of these fluids in converging and diverging channels. George Barker Jeffery and Georg Hamel were the first to study the incompressible viscous fluid flow between non-parallel walls or through convergent-divergent channels, known as the Jeffery-Hamel problem $[1,2]$. The pioneers initially introduced this type of flow and managed to give it a celebrity occupying a primary place in the field of fluid dynamics, constituting a reliable mathematical formulation for many mechanical situations encouraging many scientists to conduct extensive research in recent years, because of their use in many industrial and natural areas. The classical Jeffery-Hamel problem was further studied to include the effects of external magnetic field in the conducting fluid. The magnetic field acts as a control parameter, along with the flow, the Reynolds number, and the angle of the walls [3]. Most scientific research problems such as Jeffery-Hamel flow and other problems in fluid mechanics are inherently nonlinear. A part from a limited number of these problems, most of them do not have analytical solutions, thus the nonlinear equations are solved using other methods [4]. Jeffery-Hamel flow with high magnetic field and Nanoparticle by reconstruction of variation iteration method (RVIM) was studied by [5]. They reduced the Navier-Stokes equation and Maxwell's electromagnetism governing equations to nonlinear ordinary differential equations to model the problem. The flow field inside the divergent channel was studied with various values of Hartmann number and angle of channel. They matched their results with the exact solution obtained by Adomian's 
Decomposition Method (ADM) which were in agreement. Studies on MHD Flow of a Jeffery fluid in diverging and converging channels between oblique walls using conservation laws along with similarity transformations were done by researchers who demonstrated the effects of different parameters on the flow, [6]. They observed that magnetic field acts similar to a control parameter as it controls backflow for diverging channel and concluded that with high values of magnetic number $M$, flow separation can be controlled for diverging channels. Similarly, the flow of an incompressible viscous fluid in a converging and diverging channel was investigated by researchers and they made various conclusions from their research, [7]. For diverging channel, $\alpha>0$ the velocity decreases with the increase in angle $\alpha$ and the influence of Reynold's number $\mathrm{Re}$ and angle $\alpha$ is same for diverging channel. They noted that the effect of angle $\alpha$ is quite opposite for converging channel $(\alpha<0)$ to that for diverging channel and there is an increase in the velocity for converging channel with an increase in $\alpha$. Also for converging channel, Reynold's number Re resulted in an increase in the velocity which is opposite to that for the diverging channel.

Analytical Investigation of MHD Jeffery-Hamel nanofluid flow in non-parallel walls in 2013 was carried out by Sheikholeslami who applied the Homotopy perturbation method (HPM) to investigate the effect of magnetic field on $\mathrm{Cu}$-water nanofluid flow in non-parallel walls, [8]. The validity of HPM solutions was verified in their study by comparing their results with numerical results obtained using a fourth-order Runge-Kutta method. The results revealed that velocity in the boundary layer thickness decreased with the increase of the Reynolds number and nanoparticle volume fraction and increased with the increase in the Hartmann number. Mass and heat transfer analysis in an unsteady viscous MHD nanofluid flow through a channel with porous walls and medium in the presence of nanoparticles that were metallic were numerically studied and they discussed the two effective cases of thermal conductivity in their analysis through H-C model, [9]. They concluded that with the patronage of small values of permeability, Reynolds number and relaxation/ contraction parameter, wall contraction together with suction, flow turning is invigorated close to the wall where the boundary layer is shaped while on the other hand, when wall relaxation is coupled with injection, the flow adjacent to the porous walls decreased. A magnetohydrodynamic laminar steady two-dimensional viscous incompressible nanofluid flow from a source or sink between divergent-convergent channels in the presence of an external magnetic field vertically downward to the top wall was investigated by Alam who found that an increase in the channel semi angle leads to increase of fluid centre line velocity, an increase in the Hartmann number reduces the fluid flow in the channel centerline and produces the backward flow near the walls for both base fluid and nanofluid, the velocity increases as nanoparticles volume fraction increases along the centerline while increasing the volume fraction generates backward flow near the channel walls, [10].

In 2017, Nagler studied a Jeffery-Hamel (J-H) flow model of the non-Newtonian fluid type inside a convergent wedge (inclined walls) with wall friction. He discovered that the Newtonian normalized velocity reduces gradually with the tangential direction progress. Further, an increase in the friction coefficient leads to a reduction in the normalized Newtonian velocity profile values. He also noted that an increase in the Reynolds number causes a rise in the normalized velocity function values and for the small values of wedge semi-angle, [11]. His solutions are in agreement with the previous results obtained by other researchers.

More recently, unsteady two-dimensional Jeffery-Hamel flow of an incompressible non-Newtonian fluid, with skin friction and nonlinear viscosity, flowing through a divergent conduit in the existence of a constant magnetic field applied in the direction perpendicular to fluid motion was studied by Ochieng who noted that the fluid velocity increases with increasing values of the Reynolds number and Hartmann number, [12]. The velocity, however, decreases with increasing values of the unsteadiness parameter, and it remains constant with increasing values of the Prandtl number and Eckert number. The fluid temperature increases with increasing values of the Prandtl number, Eckert number, Reynolds number, Hartmann number, and unsteadiness parameter. In this study, analysis of unsteady MHD Jeffrey Hamel flows in the presence of an inclined magnetic field between porous walls with injection/ suction is investigated and analyzed. The aim of this study is to determine the effect of various flow parameters and variables on the unsteady Jeffrey Hamel flow in the presence of an oblique magnetic field with suction/injection.

\section{Mathematical Modeling}

In this study, considered is the unsteady two-dimensional MHD Jeffrey Hamel flow of an electrically conducting viscous incompressible fluid from a source at the intersection between two porous walls with an injection case and a suction case in the presence of an oblique variable magnetic field, where the angle between the walls is $2 \alpha$ as shown in the figure. The rigid walls are known as divergent if $\alpha>0$ and convergent if $\alpha<0$. We assume that the velocity is only along the radial direction and depends on $\mathrm{r}$ and $\theta$ so that $\mathrm{u}=$ (u $(r, \theta), 0)$.

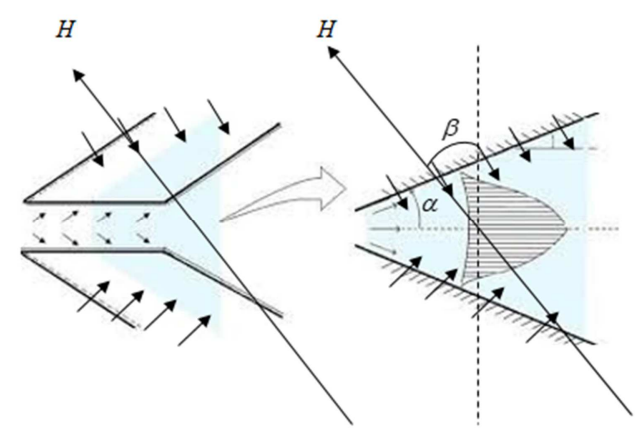

Figure 1. Geometry of the problem. 
The boundary conditions and the initial conditions for the model are such that the angle between the walls, $\theta$ is in the limits $-\alpha \leq \theta \leq \alpha$.

For $\theta=-\alpha, u_{r}=0, u_{\theta}=u_{0}, T=T_{w}, C=C_{w}$

For $\theta=0, u_{r}=u_{\infty}, u_{\theta}=0, T=T_{\infty}, C=C_{\infty}$

For $\theta=\alpha, u_{r}=0, u_{\theta}=u_{0}, T=T_{w}, C=C_{w}$

$u_{0}$ is the injection/suction velocity, $T_{\infty}$ and $T_{w}$ are the free stream and wall temperatures respectively and $T_{\infty}>T_{w}$ while $C_{\infty}$ and $C_{w}$ are the free stream and wall concentrations respectively and $C_{\infty}<C_{w}$.

\section{Governing Equations}

The general continuity equation in the cylindrical coordinate system $(r, \theta, z)$ is given by

$$
\begin{gathered}
\frac{\partial \rho}{\partial t}+\frac{1}{r} \frac{\partial}{\partial r}\left(r \rho u_{r}\right)+\frac{1}{r} \frac{\partial}{\partial \theta}\left(\rho u_{\theta}\right)+\frac{\partial}{\partial z}\left(\rho u_{z}\right)=0 \\
\rho C_{p}\left(\frac{d T}{d t}+u_{r} \frac{\partial T}{\partial r}+\frac{u_{\theta}}{r} \frac{\partial T}{\partial \theta}+u_{z} \frac{\partial T}{\partial z}\right)=\mathrm{K}\left[\frac{1}{r} \frac{\partial}{\partial r}\left(r \frac{\partial T}{\partial r}\right)+\frac{1}{r^{2}} \frac{\partial^{2} \mathrm{~T}}{\partial \theta^{2}}+\frac{\partial^{2} \mathrm{~T}}{\partial z^{2}}\right]+\Phi
\end{gathered}
$$

The motion equation in the cylindrical coordinate system along the $r$ and $\theta$ directions are given by

$$
\begin{aligned}
& \rho\left(\frac{\partial u_{r}}{\partial t}+u_{r} \frac{\partial u_{r}}{\partial r}+\frac{u_{\theta}}{r} \frac{\partial u_{r}}{\partial \theta}-\frac{u_{\theta}^{2}}{r}+u_{z} \frac{\partial u_{r}}{\partial z}\right)=-\frac{\partial p}{\partial r}+ \\
& \mu\left(\frac{1}{r} \frac{\partial}{\partial r}\left(\frac{\partial\left(r u_{r}\right)}{\partial r}\right)+\frac{1}{r^{2}}\left(\frac{\partial^{2} u_{r}}{\partial \theta^{2}}\right)-\frac{2}{r^{2}} \frac{\partial u_{\theta}}{\partial \theta}+\frac{\partial^{2} u_{r}}{\partial z^{2}}\right)+\rho F_{r} \\
& \rho\left(\frac{\partial u_{\theta}}{\partial t}+u_{r} \frac{\partial u_{\theta}}{\partial r}+\frac{u_{\theta}}{r} \frac{\partial u_{\theta}}{\partial \theta}+\frac{u_{r} u_{\theta}}{r}+u_{z} \frac{\partial u_{\theta}}{\partial z}\right)=-\frac{1}{r} \frac{\partial p}{\partial \theta}+ \\
& \mu\left(\frac{1}{r} \frac{\partial}{\partial r}\left(\frac{\partial\left(r u_{\theta}\right)}{\partial r}\right)+\frac{1}{r^{2}}\left(\frac{\partial^{2} u_{\theta}}{\partial \theta^{2}}\right)+\frac{2}{r^{2}} \frac{\partial u_{r}}{\partial \theta}+\frac{\partial^{2} u_{\theta}}{\partial z^{2}}\right)+\rho F_{\theta}
\end{aligned}
$$

The conservation of energy equation derived from the first law of thermodynamics in the cylindrical coordinate system is given by,
The equation of species concentration is based on the law of conservation of mass.

$$
\frac{\partial C}{\partial t}+u_{r} \frac{\partial C}{\partial r}+u_{\theta} \frac{\partial C}{\partial \theta}=D\left(\frac{1}{r} \frac{\partial C}{\partial r}+\frac{\partial^{2} C}{\partial r^{2}}+\frac{1}{r^{2}} \frac{\partial^{2} C}{\partial \theta^{2}}\right)
$$

This is the induction equation in vector form which describes how the magnetic fields in a perfectly conducting fluid changes with time under the influence of a velocity $\mathbf{V}$.

$$
\frac{\partial \mathbf{H}}{\partial t}=\frac{1}{\mu_{e} \sigma}\left(\nabla^{2} \mathbf{H}\right)+\frac{1}{\mu_{e}}(\nabla \times[\mathbf{V} \times \mathbf{B}])
$$

The Lorentz force is a force due to the combination of the electric and the magnetic force on a point charge due to electromagnetic fields. The total electromagnetic force is given by $\mathbf{J} \times \mathbf{B}$. From Ohms law, the total electric field $\mathbf{J}$ is given by

$$
\mathbf{J}=\sigma(\mathbf{E}+\mathbf{V} \times \mathbf{B})
$$

Neglecting $\mathbf{E}$, the total electric field is given by

$$
\mathbf{J}=\sigma(\mathbf{V} \times \mathbf{B})
$$

The velocity is given by $\mathbf{V}=u_{r} \cos \theta \hat{r}+0 \hat{\theta}+0 \hat{z}$, the inclined variable magnetic field $\mathbf{H}_{1}=-H \sin \beta \hat{r}+H \cos \beta \hat{\theta}+0 \hat{z}$ and the induced magnetic field $\mathbf{H}=H_{r} \hat{r}+H_{\theta} \hat{\theta}+0 \hat{z}$.

Therefore the total magnetic field $\mathbf{B}$ is given by $\mathbf{B}=\mathbf{H}_{1}+\mathbf{H}$ hence $\mathbf{B}$ is given by

$$
\mathbf{B}=\left(H_{r}-H \sin \beta\right) \hat{r}+\left(H_{\theta}+H \cos \beta\right) \hat{\theta}+0 \hat{z}
$$

From equation (3.4.1.3) which simplifies to:

$$
\mathbf{V} \times \mathbf{B}=u_{r} \sigma\left(H_{\theta}+H \cos \beta\right) \cos \theta \hat{z}
$$

$$
\nabla \times[\mathbf{V} \times \mathbf{B}]=\left|\begin{array}{ccc}
\hat{r} & \hat{\theta} & \hat{z} \\
\frac{1}{r} \frac{\partial}{\partial r}(r) & \frac{1}{r} \frac{\partial}{\partial \theta} & \frac{\partial}{\partial z} \\
0 & 0 & u_{r} \sigma\left(H_{\theta}+H \cos \beta\right) \cos \theta
\end{array}\right|
$$

$$
\begin{aligned}
& \nabla \times[\mathbf{V} \times \mathbf{B}]=\frac{1}{r} \frac{\partial}{\partial \theta}\left[u_{r} \sigma\left(H_{\theta}+H \cos \beta\right) \cos \theta\right] \hat{r}+ \\
& \frac{1}{r} \frac{\partial}{\partial r}\left[r u_{r} \sigma\left(H_{\theta}+H \cos \beta\right) \cos \theta\right] \hat{\theta}
\end{aligned}
$$

The conservation of energy equation derived from the first law of thermodynamics in the cylindrical coordinate system is given by the specific governing equations for the fluid flow that is purely radial and depends on $r$ and $\theta$ with no change in the flow parameters along the $z$ direction using the Boussinesq approximation, considering the Joule heating and the viscous dissipation with no chemical reaction are obtained as;

$$
\frac{1}{r} \frac{\partial}{\partial r}\left(r u_{r}(r, \theta)\right)=0
$$




$$
\begin{aligned}
& \hat{r}: \rho\left(\frac{\partial u_{r}}{\partial t}+u_{r} \frac{\partial u_{r}}{\partial r}-\frac{u_{\theta}^{2}}{r}+\frac{u_{\theta}}{r} \frac{\partial u_{r}}{\partial \theta}\right)=-\frac{\partial p}{\partial r}+\mu\left(\frac{1}{r} \frac{\partial}{\partial r}\left(\frac{\partial\left(r u_{r}\right)}{\partial r}\right)+\frac{1}{r^{2}}\left(\frac{\partial^{2} u_{r}}{\partial \theta^{2}}\right)\right) \\
& -\left(u_{r} \sigma\left(H_{\theta}+H \cos \beta\right)^{2} \cos \theta\right) \\
& \hat{\theta}: \rho\left(\frac{\partial u_{\theta}}{\partial t}+u_{r} \frac{\partial u_{\theta}}{\partial r}+\frac{u_{r} u_{\theta}}{r}\right)=-\frac{1}{r} \frac{\partial p}{\partial \theta}+\mu\left(\frac{1}{r} \frac{\partial}{\partial r}\left(\frac{\partial\left(r u_{\theta}\right)}{\partial r}\right)+\frac{2}{r^{2}} \frac{\partial u_{r}}{\partial \theta}\right)+ \\
& \left(u_{r} \sigma\left(H_{r}-H \sin \beta\right)\left(H_{\theta}+H \cos \beta\right) \cos \theta\right)+\rho g\left[\beta_{*}\left(T-T_{\infty}\right)+\beta_{c}\left(C-C_{\infty}\right)\right] \\
& \rho C_{p}\left(\frac{d T}{d t}+u_{r} \frac{\partial T}{\partial r}+\frac{u_{\theta}}{r} \frac{\partial T}{\partial \theta}\right)=\mathrm{K}\left(\frac{1}{r} \frac{\partial T}{\partial r}+\frac{\partial^{2} T}{\partial r^{2}}+\frac{1}{r^{2}} \frac{\partial^{2} \mathrm{~T}}{\partial \theta^{2}}\right)+ \\
& \mu\left[2\left(\frac{\partial u_{r}}{\partial r}\right)^{2}+2\left(\frac{u_{r}}{r}\right)^{2}+\left(-\frac{u_{\theta}}{r}\right)^{2}-\frac{2 u_{\theta}}{r^{2}} \cdot\left(\frac{\partial u_{r}}{\partial \theta}\right)+\frac{1}{r^{2}}\left(\frac{\partial u_{r}}{\partial \theta}\right)^{2}\right]+ \\
& \sigma\left(u_{r}\left(H_{\theta}+H \cos \beta\right) \cos \theta\right)^{2} \\
& \frac{\partial C}{\partial t}+u_{r} \frac{\partial C}{\partial r}+u_{\theta} \frac{\partial C}{\partial \theta}=D\left(\frac{1}{r} \frac{\partial C}{\partial r}+\frac{\partial^{2} C}{\partial r^{2}}+\frac{1}{r^{2}} \frac{\partial^{2} C}{\partial \theta^{2}}\right) \\
& \hat{r}: \frac{\partial H_{r}}{\partial t}=\frac{1}{\mu_{e} \sigma}\left(\frac{\partial^{2} H_{r}}{\partial r^{2}}+\frac{1}{r} \frac{\partial H_{r}}{\partial r}+\frac{1}{r^{2}} \frac{\partial^{2} H_{r}}{\partial \theta^{2}}\right)-\frac{u_{r} \sigma}{r} \frac{1}{\mu_{e}}\left(H_{\theta} \sin \theta+H \cos \beta \sin \theta\right) \\
& \hat{\theta}: \frac{\partial H_{\theta}}{\partial t}=\frac{1}{\mu_{e} \sigma}\left(\frac{\partial^{2} H_{\theta}}{\partial r^{2}}+\frac{1}{r} \frac{\partial H_{\theta}}{\partial r}+\frac{1}{r^{2}} \frac{\partial^{2} H_{\theta}}{\partial \theta^{2}}\right)+ \\
& \frac{\sigma}{\mu_{e}}\left[\frac{\partial u_{r}}{\partial r}\left(H_{\theta}+H \cos \beta\right) \cos \theta+\frac{u_{r}}{r}\left(H_{\theta}+H \cos \beta\right) \cos \theta\right]
\end{aligned}
$$

Differentiating (12) with respect to $\hat{\theta}$, expanding and Multiplying (13) by $\hat{r}$ and differentiating with respect to $\hat{r}$ and getting the difference in the two equations to obtain the equation of momentum in cylindrical form.

$$
\begin{aligned}
& \frac{\partial^{2} u_{r}}{\partial t \partial \theta}+\frac{\partial u_{r}}{\partial \theta} \cdot \frac{\partial u_{r}}{\partial r}+u_{r} \frac{\partial^{2} u_{r}}{\partial \theta \partial r}+\frac{u_{\theta}}{r} \frac{\partial^{2} u_{r}}{\partial \theta^{2}}-u_{\theta} \frac{\partial u_{r}}{\partial r}= \\
& \frac{\mu}{\rho}\left(\frac{1}{r} \frac{\partial}{\partial \theta}\left(\frac{\partial u_{r}}{\partial r}\right)-\frac{u_{r}}{r^{2}}+\frac{\partial}{\partial \theta}\left(\frac{\partial^{2} u_{r}}{\partial r^{2}}\right)+\frac{1}{r^{2}}\left(\frac{\partial^{3} u_{r}}{\partial \theta^{3}}\right)-\frac{u_{\theta}}{r^{2}}+\frac{2}{r^{2}} \frac{\partial u_{r}}{\partial \theta}-\frac{2}{r} \frac{\partial}{\partial \theta}\left(\frac{\partial u_{r}}{\partial r}\right)\right) \\
& +u_{r}\left(\frac{1}{\rho} \sigma\left(H_{\theta}+H \cos \beta\right)^{2} \sin \theta\right)-\frac{\partial u_{r}}{\partial \theta}\left(\frac{1}{\rho} \sigma\left(H_{\theta}+H \cos \beta\right)^{2} \cos \theta\right)- \\
& \left(u_{r}+r \frac{\partial u_{r}}{\partial r}\right) \frac{\sigma}{\rho}\left(\left(H_{r}-H \sin \beta\right)\left(H_{\theta}+H \cos \beta\right) \cos \theta\right)-g\left[\beta_{*}\left(T-T_{\infty}\right)+\beta_{c}\left(C-C_{\infty}\right)\right]
\end{aligned}
$$

Equations (11), (13), (15), (16), (17) and (18) are the final set of the equations governing the flow.

\section{Unsteadiness Parameter}

The unsteadiness parameter is introduced to account for unsteadiness of the fluid flow. The unsteadiness parameter is a constant that represents the dimensionless measure of unsteadiness.

According to $[11,13-15]$, the unsteadiness parameter is defined by

$$
\lambda=\frac{\delta^{m}}{v r^{m-1}} \frac{d \delta}{d t}
$$


where $\delta(t)$ is a time-dependent length scale, $m$ is a parameter related to the wedge angle and the radius of the wedge.

\section{Wedge angle Parameter}

The wedge angle parameter is an arbitrary constant $m$ related to the wedge angle, [13]. From the studies by [15, $10]$, the researchers concluded that if the total angle of the wedge is $\Omega$, then the wedge angle parameter is given by the relation

$$
\Omega=\frac{2 m}{m+1} \pi
$$

But from the geometry in Figure $1, \Omega=2 \alpha$, hence

$$
2 \alpha=\frac{2 m}{m+1} \pi
$$

The relationship between $\alpha$ and the $m$ related to the wedge angle is given by

$$
\alpha=\frac{m}{m+1} \pi
$$

\section{Similarity Transformation}

The equations governing the flow are subjected to a similarity transformation to reduce them to ordinary differential equations before being solved numerically.

$$
\begin{aligned}
& r^{2} \frac{m+1}{\delta v} \frac{d \delta}{d t} f^{\prime}-2 Q \frac{1}{v \delta^{m+1}} f f^{\prime}-\frac{r u_{\theta}}{v}\left(f^{\prime \prime}+f\right)=f-5 f^{\prime}-f^{\prime \prime \prime} \\
& -\frac{r u_{\theta} \delta^{m+1}}{Q}-r^{2} f \frac{\sigma}{\mu}\left(H_{\theta}+H \cos \beta\right)^{2} \sin \theta+r^{2} f^{\prime} \frac{\sigma}{\mu}\left(H_{\theta}+H \cos \beta\right)^{2} \cos \theta \\
& -g \frac{\beta_{*} r^{3}\left(T_{w}-T_{\infty}\right) \omega}{v Q}-g \frac{\beta_{c} r^{3}\left(C-C_{\infty}\right) \phi}{v Q}
\end{aligned}
$$

Introducing the dimensionless numbers

$$
R_{e}=\frac{r u_{\theta}}{v}=\frac{Q}{v r}, H_{a}=r^{2} \frac{\sigma}{\mu}\left(H_{\theta}+H \cos \beta\right)^{2}, G_{r(T)}=\frac{g \beta_{*} r^{3}\left(T_{w}-T_{\infty}\right)}{v Q}, G_{r(C)}=\frac{g \beta_{c} r^{3}\left(C-C_{\infty}\right)}{v Q}
$$

in equation (26)

$$
\begin{aligned}
& (m+1) \frac{\delta^{m+1}}{r^{m+1}} \lambda f^{\prime}-2 r R_{e} \frac{1}{\delta^{m+1}} f f^{\prime}-R_{e}\left(f^{\prime \prime}+f\right)=f-5 f^{\prime}-f^{\prime \prime \prime} \\
& -\frac{r u_{\theta} \delta^{m+1}}{Q}-H_{a}^{2} f \sin \theta+H_{a}^{2} f^{\prime} \cos \theta-G_{r(T)} \omega-G_{r(C)} \phi
\end{aligned}
$$

Rearranging equation (27)

$$
\begin{aligned}
& f^{\prime \prime \prime}=R_{e}\left(f^{\prime \prime}+f\right)+2 r R_{e} \frac{1}{\delta^{m+1}} f f^{\prime}+f-5 f^{\prime}-\frac{r u_{\theta} \delta^{m+1}}{Q}- \\
& H_{a}^{2} f \sin \theta+H_{a}^{2} f^{\prime} \cos \theta-G_{r(T)} \omega-G_{r(C)} \phi-(m+1) \frac{\delta^{m+1}}{r^{m+1}} \lambda f^{\prime}
\end{aligned}
$$


Equation (28) is the final equation of momentum

Using the similarity transformations on equation (13)

$$
\begin{aligned}
& \rho C_{p}\left(-\frac{m+1}{\delta^{m+2}} \frac{d \delta}{d t}\left(T_{w}-T_{\infty}\right) \omega+\frac{u_{\theta}}{r} \cdot \frac{T_{w}-T_{\infty}}{\delta^{m+1}} \omega^{\prime}\right)=\mathrm{K}\left(\frac{1}{r^{2}} \cdot \frac{T_{w}-T_{\infty}}{\delta^{m+1}} \omega^{\prime \prime}\right)+ \\
& \mu\left[2\left(\frac{Q}{r^{2}} \frac{1}{\delta^{m+1}} f\right)^{2}+2\left(-\frac{Q}{r^{2}} \frac{1}{\delta^{m+1}} f\right)^{2}+\left(-\frac{u_{\theta}}{r}\right)^{2}-\frac{2 u_{\theta}}{r^{2}} \cdot\left(-\frac{Q}{r} \frac{1}{\delta^{m+1}} f^{\prime}\right)+\frac{1}{r^{2}}\left(-\frac{Q}{r} \frac{1}{\delta^{m+1}} f^{\prime}\right)^{2}\right]+ \\
& \sigma\left(\left(-\frac{Q}{r} \frac{1}{\delta^{m+1}} f\right)\left(H_{\theta}+H \cos \beta\right) \cos \theta\right)^{2}
\end{aligned}
$$

Introducing the unsteadiness parameter to the equation (29)

$$
\begin{aligned}
& -\frac{m+1}{\delta^{m+1}} r^{m-1} v \lambda \omega+\frac{u_{\theta}}{r} \omega^{\prime}=\frac{\mathrm{K}}{\rho C_{p} r^{2}} \omega^{\prime \prime}+ \\
& \frac{Q^{2} \mu}{r^{2} \rho C_{p}\left(T_{w}-T_{\infty}\right)}\left[\frac{4}{r^{2}} \frac{1}{\delta^{m+1}} f^{2}+\frac{u_{\theta}^{2}}{Q^{2}} \delta^{m+1}+\frac{2 u_{\theta}}{r Q} f^{\prime}+\frac{1}{r^{2}} \frac{1}{\delta^{m+1}} f^{\prime 2}\right]+ \\
& \frac{\sigma Q^{2}}{\rho C_{p} r^{2}\left(T_{w}-T_{\infty}\right)} \frac{1}{\delta^{m+1}} f^{2}\left(\left(H_{\theta}+H \cos \beta\right) \cos \theta\right)^{2}
\end{aligned}
$$

Introducing the dimensionless numbers

$$
P_{r}=\frac{\mu C_{p}}{\mathrm{~K}}, R_{e}=\frac{r \cdot u_{\theta}}{v}, E_{c}=\frac{Q^{2}}{r^{2} C_{p}\left(T_{w}-T_{\infty}\right)}, J=\frac{\sigma\left(H_{\theta}+H \cos \beta\right)^{2}}{\rho C_{p} v\left(T_{w}-T_{\infty}\right)}
$$

Equation (30) becomes

$$
\begin{aligned}
& -\frac{m+1}{\delta^{m+1}} r^{m-1} \lambda \omega+\frac{1}{r^{2}} R_{e} \omega^{\prime}=\frac{1}{r^{2} P_{r}} \omega^{\prime \prime}+E_{c}\left[\frac{4}{r^{2}} \frac{1}{\delta^{m+1}} f^{2}+\frac{u_{\theta}^{2}}{Q^{2}} \delta^{m+1}+\frac{2 u_{\theta}}{r Q} f^{\prime}+\frac{1}{r^{2}} \frac{1}{\delta^{m+1}} f^{\prime 2}\right]+ \\
& \frac{Q^{2}}{r^{2}} J \frac{1}{\delta^{m+1}} f^{2}\left(\cos ^{2} \theta\right)
\end{aligned}
$$

Simplifying and rearranging equation (31), the energy equation is obtained as;

$$
\begin{aligned}
& \omega^{\prime \prime}=-\frac{m+1}{\delta^{m+1}} r^{m+1} P_{r} \lambda \omega+P_{r} R_{e} \omega^{\prime}-P_{r} \cdot E_{c}\left[4 \frac{1}{\delta^{m+1}} f^{2}+\frac{r^{2} u_{\theta}^{2}}{Q^{2}} \delta^{m+1}+\frac{2 r u_{\theta}}{Q} f^{\prime}+\frac{1}{\delta^{m+1}} f^{\prime 2}\right] \\
& -Q^{2} P_{r} J \frac{1}{\delta^{m+1}} f^{2}\left(\cos ^{2} \theta\right)
\end{aligned}
$$

Using the similarity transformation on equation (15)

$$
-\frac{m+1}{\delta^{m+2}} \frac{d \delta}{d t}\left(C_{w}-C_{\infty}\right) \phi+\left(-\frac{Q}{r} \frac{1}{\delta^{m+1}} f\right)(0)+u_{\theta} \frac{C_{w}-C_{\infty}}{\delta^{m+1}} \phi^{\prime}=D\left(\frac{1}{r}(0)+(0)+\frac{1}{r^{2}} \frac{C_{w}-C_{\infty}}{\delta^{m+1}} \phi^{\prime \prime}\right)
$$

Simplifying equation (33)

$$
-\frac{m+1}{\delta^{m+2}} \frac{d \delta}{d t}\left(C_{w}-C_{\infty}\right) \phi+u_{\theta} \frac{C_{w}-C_{\infty}}{\delta^{m+1}} \phi^{\prime}=D\left(\frac{1}{r^{2}} \frac{C_{w}-C_{\infty}}{\delta^{m+1}} \phi^{\prime \prime}\right)
$$

Introducing the dimensionless numbers

$$
R_{e}=\frac{r \cdot u_{\theta}}{v}, S_{c}=\frac{v}{D}
$$




$$
-\frac{m+1}{\delta^{m+1}} r^{m+1} \lambda \phi+r \mathrm{R}_{\mathrm{e}} \phi^{\prime}-\frac{1}{S_{c}} \phi^{\prime \prime}=0
$$

Rearranging equation (35), the equation of concentration after the transformation becomes;

$$
\phi^{\prime \prime}=r \mathrm{R}_{\mathrm{e}} \cdot S_{c} \phi^{\prime}-\frac{m+1}{\delta^{m+1}} r^{m+1} \lambda . S_{c} \phi
$$

Using the similarity transformations on equation (16) and (17). Equation (17) reduces to zero while equation (16) becomes

$$
\begin{aligned}
& -\frac{m+1}{\delta^{m+2}} \frac{Q}{r} \frac{d \delta}{d t} H=\frac{1}{\mu_{e} \sigma}\left(-\frac{2 Q}{r^{3}} \frac{1}{\delta^{m+1}} H+\frac{Q}{r^{3}} \frac{1}{\delta^{m+1}} H-\frac{Q}{r^{3}} \frac{1}{\delta^{m+1}} H^{\prime \prime}\right)+ \\
& \frac{Q}{r^{2}} \frac{1}{\delta^{m+1}} f \frac{\sigma}{\mu_{e}}\left(H_{\theta}+H \cos \beta\right) \sin \theta
\end{aligned}
$$

Introducing the unsteadiness parameter and the dimensionless parameters

$$
R_{e}=\frac{r u_{\theta}}{v}, R_{m}=\sigma \mu_{e} r u_{\theta}, H_{a}=r\left(\frac{\sigma}{\mu_{e}}\right)^{1 / 2}\left(H_{\theta}+H \cos \beta\right), \sqrt{\left(\frac{\mu_{e}}{\sigma}\right)} H_{a}=r\left(H_{\theta}+H \cos \beta\right)
$$

Equation (37) reduces to

$$
-r^{m+2} \frac{m+1}{\delta^{m+1}} \lambda H=-\frac{R_{e}}{R_{m}}\left(H+H^{\prime \prime}\right)+\frac{\sigma}{\mu_{e} v} \sqrt{\left(\frac{\mu_{e}}{\sigma}\right)} H_{a} f \sin \theta
$$

Simplifying equation (38)

$$
-r^{m+2} \frac{m+1}{\delta^{m+1}} \lambda H=-\frac{R_{e}}{R_{m}}\left(H+H^{\prime \prime}\right)+\frac{1}{v} \sqrt{\left(\frac{\sigma}{\mu_{e}}\right)} H_{a} f \sin \theta
$$

Rearranging equation (39), the final induction equation after the transformation is obtained as;

$$
H^{\prime \prime}=r^{m+2} \frac{m+1}{\delta^{m+1}} \lambda \frac{R_{m}}{R_{e}} H-H+\frac{1}{v} \sqrt{\left(\frac{\sigma}{\mu_{e}}\right)} H_{a} \frac{R_{m}}{R_{e}} f \sin \theta
$$

Equations (28), (32), (36) and (40) are the final set of ODEs of higher-order governing the fluid flow. The boundary conditions are transformed using the similarity transforms (22) to (25) to obtain For

$$
\theta=0, f(0)=-\frac{u_{\infty} r}{Q} \delta^{m+1}, u_{\theta}=0, w(0)=0, \phi(0)=0
$$

For

$$
\theta= \pm \alpha, f( \pm \alpha)=0, u_{\theta}=u_{0}, w( \pm \alpha)=\delta^{m+1}, \phi(0)=\delta^{m+1}
$$

\section{Reduction of Order of ODEs}

The equations governing the flow (28), (32), (36) and (40) are reduced to first-order ODEs and solved by the collocation method. By letting

$$
y_{1}=f, y_{2}=f^{\prime}, y_{3}=f^{\prime \prime}, y_{4}=\omega, y_{5}=\omega^{\prime}, y_{6}=\phi, y_{7}=\phi^{\prime}, y_{8}=H, y_{9}=H^{\prime}
$$

Differentiating (42) 


$$
\begin{gathered}
y_{1}^{\prime}=y_{2} \\
y_{2}^{\prime}=y_{3} \\
y_{3}^{\prime}=f^{\prime \prime \prime}=R_{e}\left(y_{3}+y_{1}\right)+2 r R_{e} \frac{1}{\delta^{m+1}} y_{1} y_{2}+y_{1}-5 y_{2}-\frac{r u_{\theta} \delta^{m+1}}{Q}- \\
H_{a}^{2} y_{1} \sin \theta+H_{a}^{2} y_{2} \cos \theta-G_{r(T)} \omega-G_{r(C)} \phi-(m+1) \frac{\delta^{m+1}}{r^{m+1}} \lambda y_{2} \quad(A) \\
y_{4}^{\prime}=y_{5} \\
y_{5}^{\prime}=\omega^{\prime \prime}=-\frac{m+1}{\delta^{m+1}} r_{r}^{m+1} P_{r} \lambda y_{4}+P_{r} R_{e} y_{5}-P_{r} \cdot E_{c}\left[4 \frac{1}{\delta^{m+1}} y_{1}^{2}\left(\cos ^{2} \theta\right) \quad y_{1}^{2}+\frac{r^{2} u_{\theta}^{2}}{Q^{2}} \delta^{m+1}+\frac{2 r u_{\theta}}{Q} y_{2}+\frac{1}{\delta^{m+1}} y_{2}{ }^{2}\right] \\
(B) \quad y_{6}^{\prime}=y_{7} \\
y_{7}^{\prime}=\phi^{\prime \prime}=r \mathrm{R}_{\mathrm{e}} \cdot S_{c} y_{7}-\frac{m+1}{\delta^{m+1}} r^{m+1} \lambda \cdot S_{c} y_{6}(C) \\
y_{8}^{\prime}=y_{9} \\
y_{9}^{\prime}=H^{\prime \prime}=r^{m+2} \frac{m+1}{\delta^{m+1}} \lambda \frac{R_{m}}{R_{e}} y_{8}-y_{8}+\frac{1}{v} \sqrt{\left(\frac{\sigma}{\mu_{e}}\right)} H_{a} \frac{R_{m}}{R_{e}} y_{1} \sin \theta \quad(D)
\end{gathered}
$$

Equations (43) to (51) become a system of equations of the general form

$$
y^{\prime}=F(\theta, y)
$$

Where

$$
y=\left(\begin{array}{l}
y_{1} \\
y_{2} \\
y_{3} \\
y_{4} \\
y_{5} \\
y_{6} \\
y_{7} \\
y_{8} \\
y_{9}
\end{array}\right)
$$

and

$$
F=\left(\begin{array}{l}
y_{2} \\
y_{3} \\
A \\
y_{5} \\
B \\
y_{7} \\
C \\
y_{9} \\
D
\end{array}\right)
$$

The system of equations (53) is solved by the collocation method using the inbuilt MATLAB function bvp4c that implements the collocation algorithm together with the implicit Runge-Kutta method.

\section{Results and Discussion}

The research findings and the discussions are presented with the graphical illustrations of the effects of various parameters on the unsteady magnetohydrodynamic JeffreyHamel flow in the presence of a variable inclined magnetic field with suction and injection. 


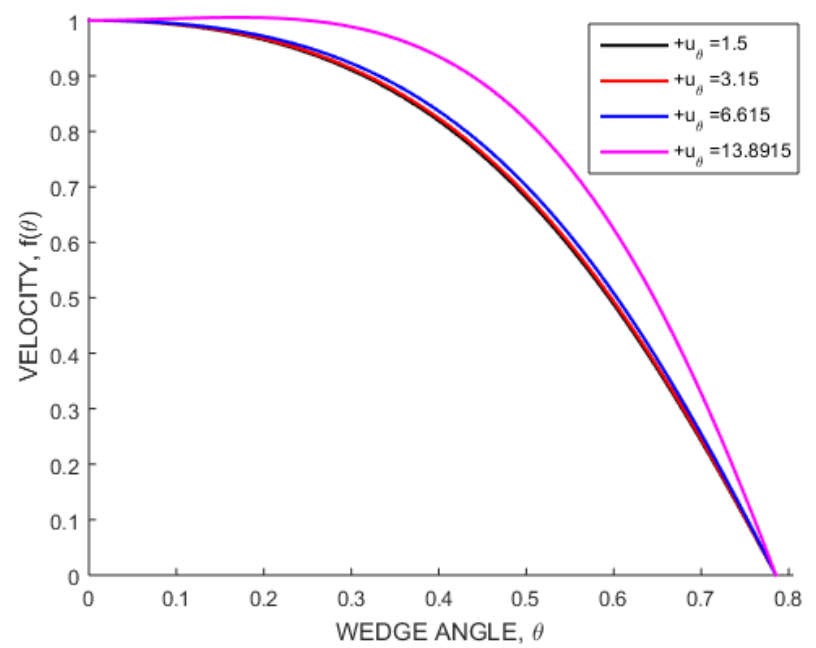

Figure 2. Velocity profiles for different values of the suction parameter.

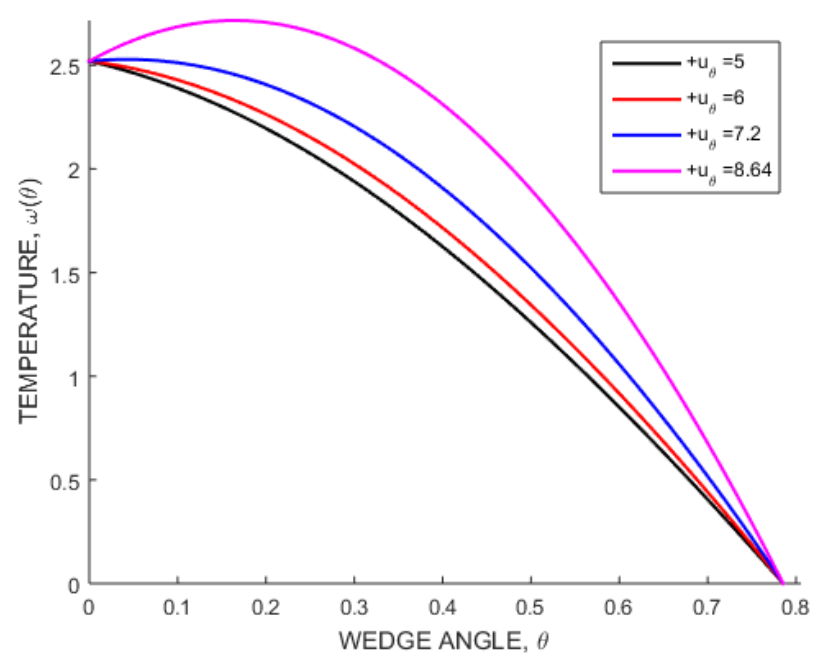

Figure 3. Temperature profiles for different values of the suction parameter.

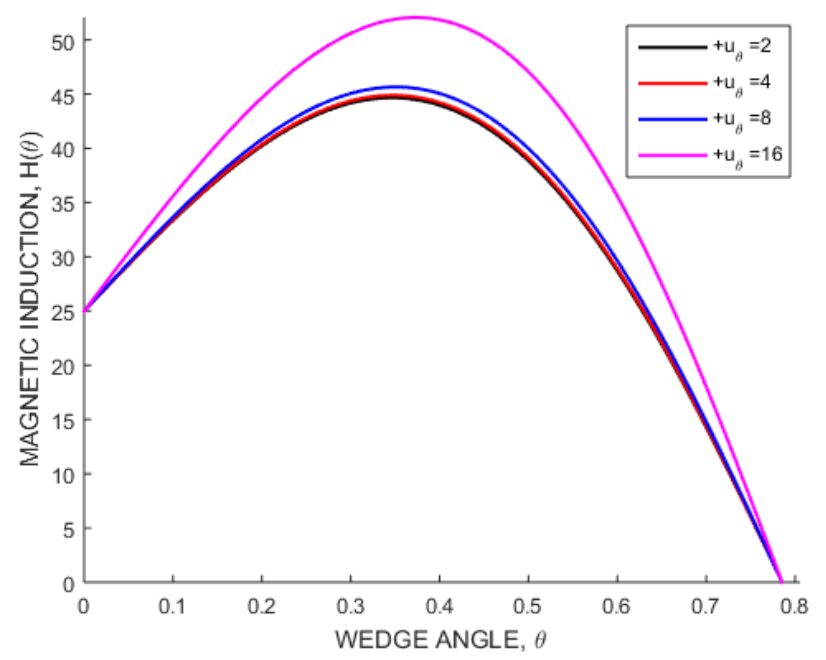

Figure 4. Magnetic Induction profiles for different values of the suction parameter.

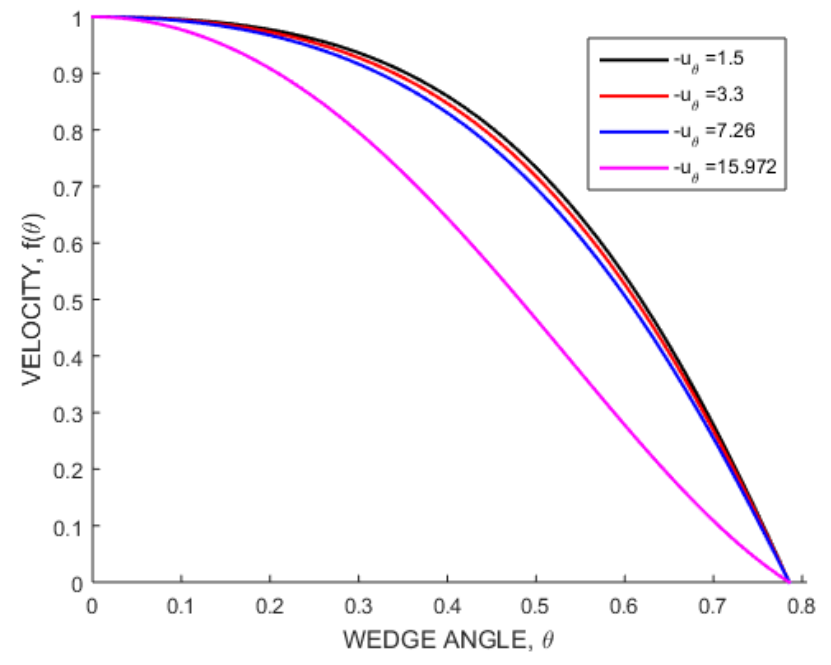

Figure 5. Velocity profiles for different values of the injection parameter.

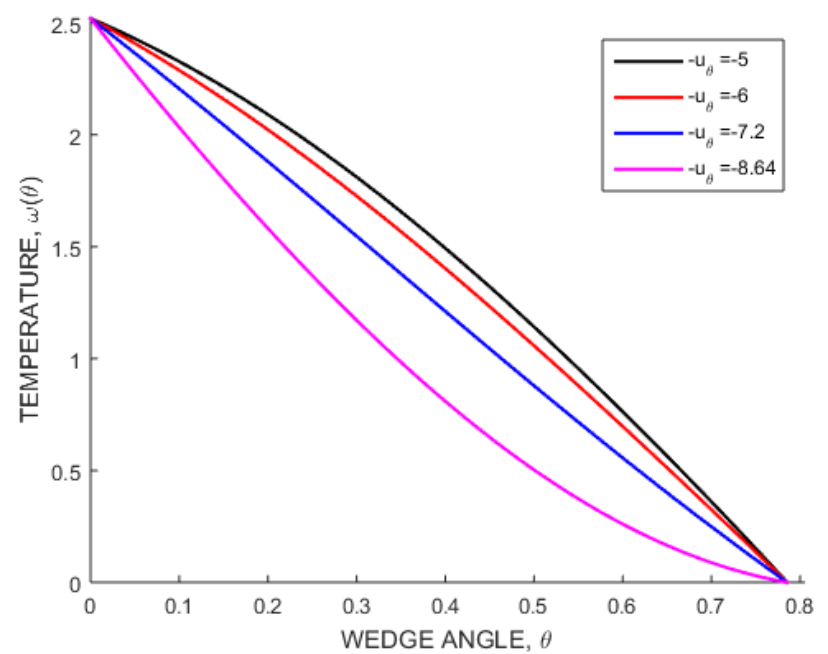

Figure 6. Temperature profiles for different values of the injection parameter.

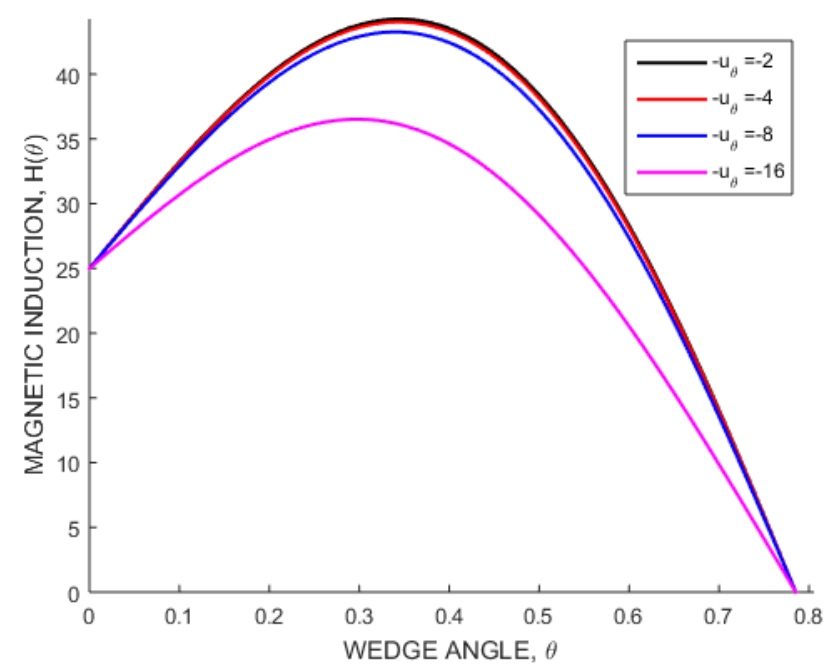

Figure 7. Velocity profiles for different values of the injection parameter. 


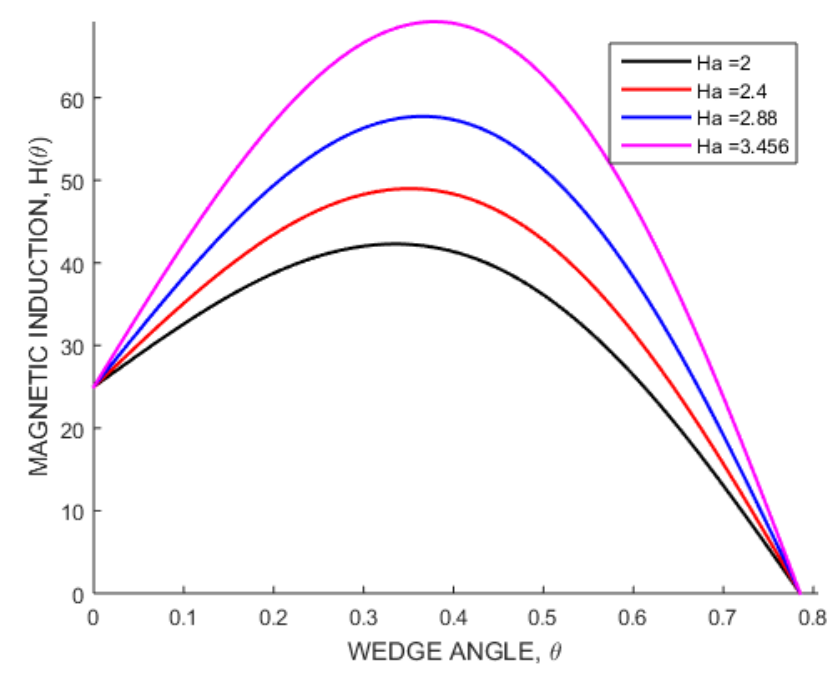

Figure 8. Magnetic Induction profiles for different values of the Hartmann number.

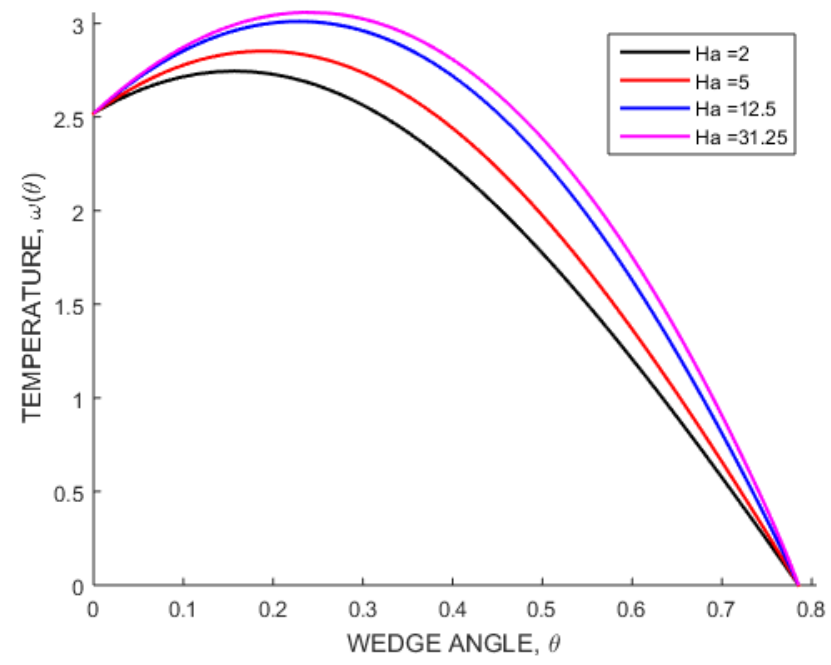

Figure 9. Temperature profiles for different values of the Hartmann number.

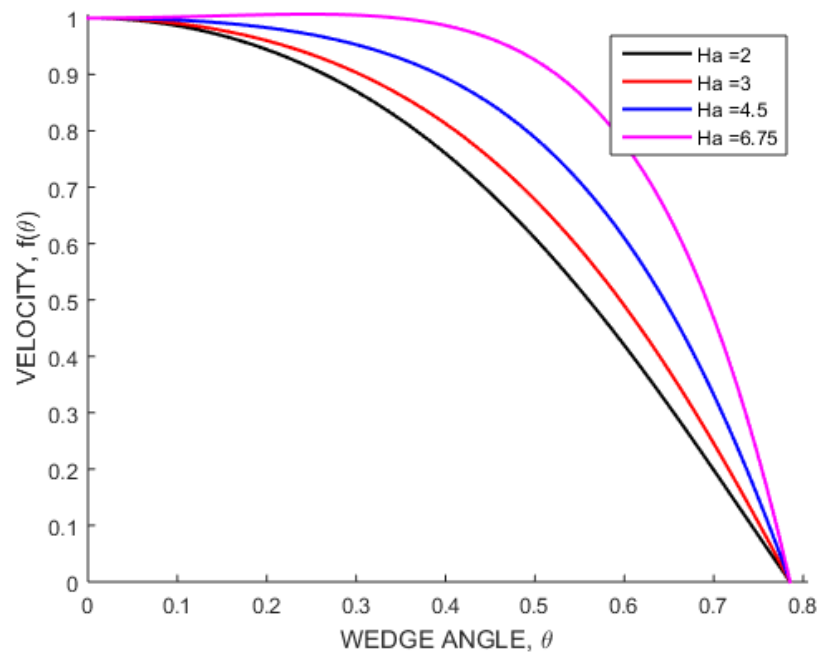

Figure 10. Velocity profiles for different values of the Hartmann number.

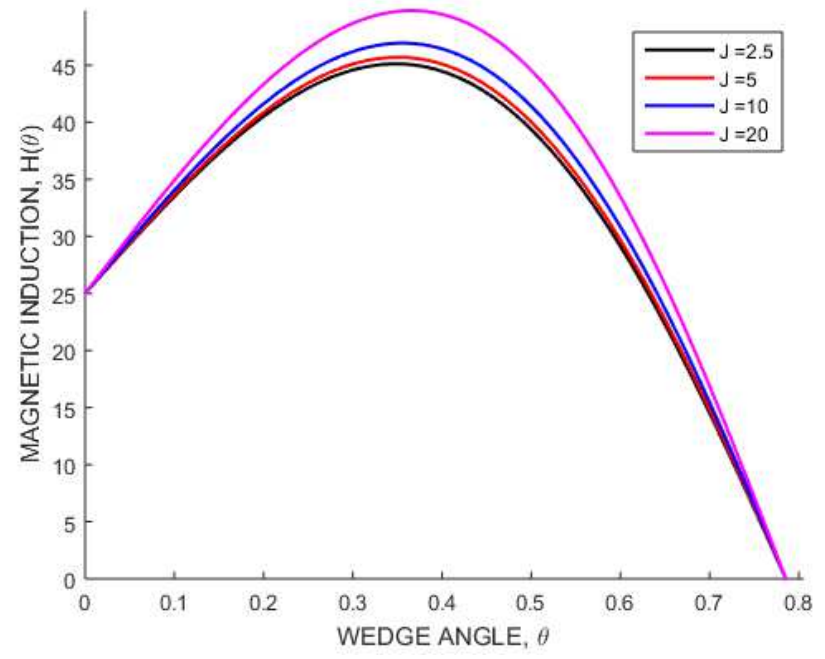

Figure 11. Magnetic Induction profiles for different values of the Joule parameter.

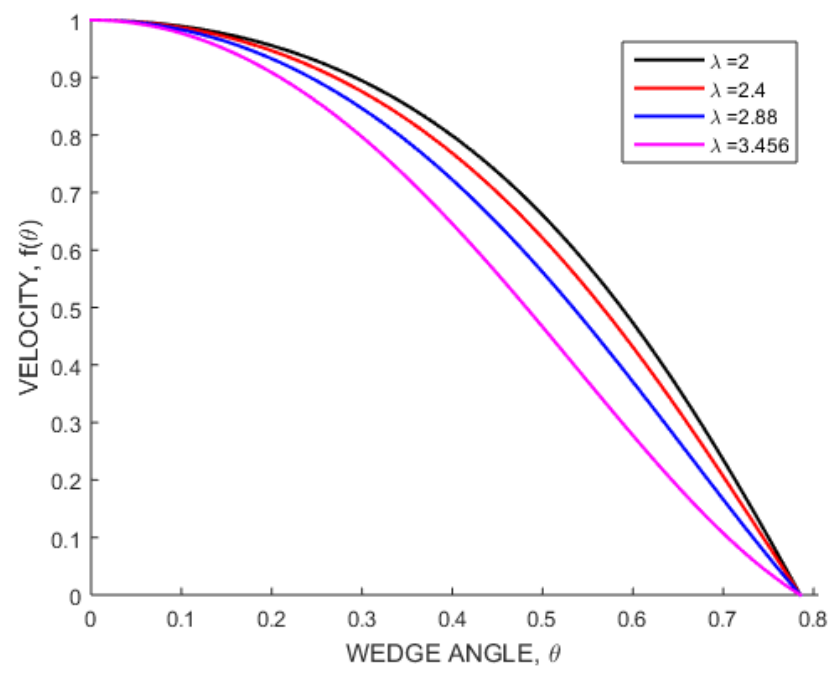

Figure 12. Velocity profiles for different values of the unsteadiness parameter.

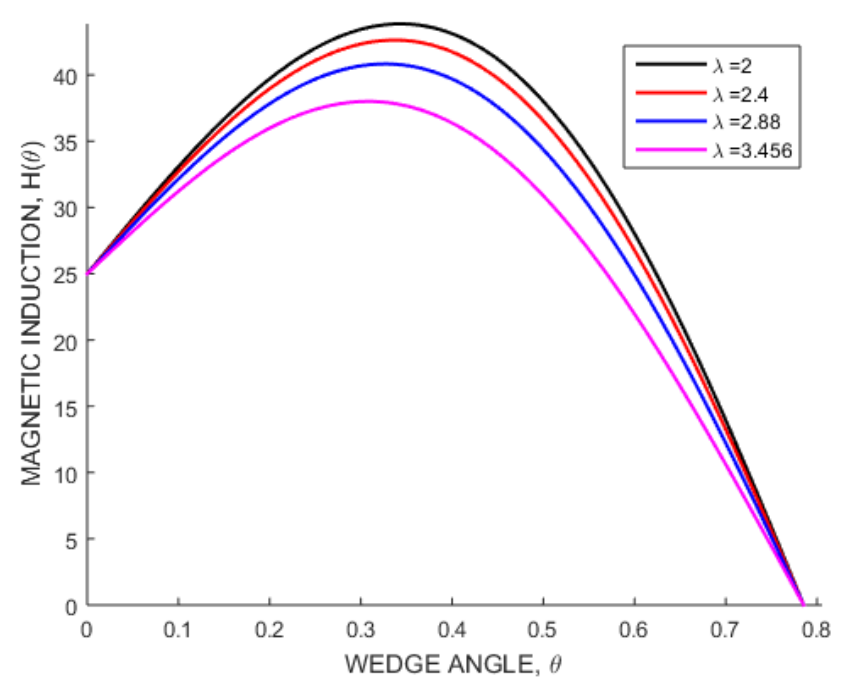

Figure 13. Magnetic induction profiles for different values of the unsteadiness parameter. 


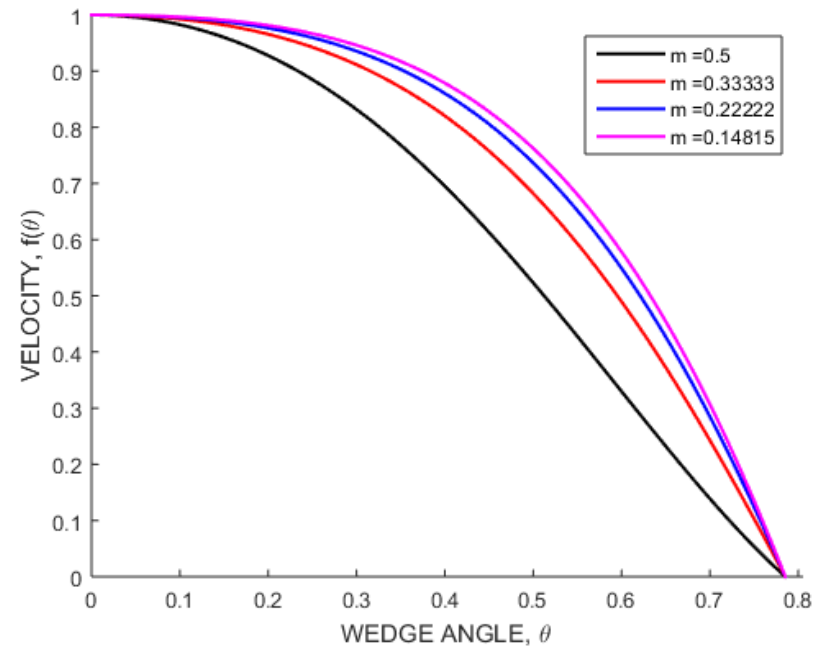

Figure 14. Velocity profiles for different values of the wedge angle.

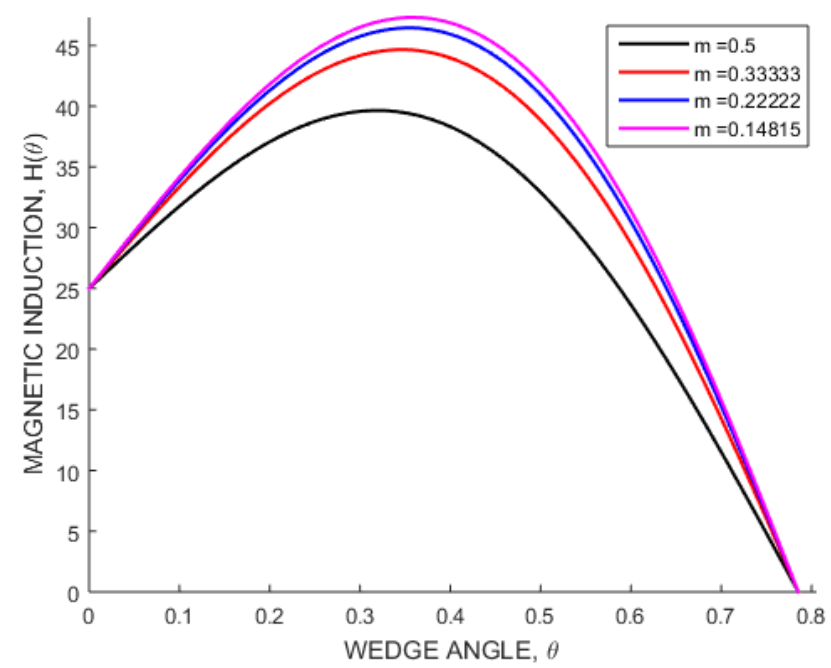

Figure 15. Magnetic induction profiles for different values of the wedge angle.

From Figure 2, the graph of the velocity profiles while varying the suction parameter, the velocity increases with the increase in the suction parameter. Suction results in the thinning of the momentum boundary layer of the fluid flow, decrease in pressure which leads to increase the velocities.

From Figure 3, the graph of the temperature profiles while varying the suction parameter, the temperature increases with the increase in the suction parameter. This is due to the increase in the velocities as a result of the suction which leads to an increase in the kinetic energy which is then converted into thermal energy hence the increase in temperature.

From Figure 4, the magnetic induction increases with the increase in the suction parameter. With the increase in the suction parameter, the velocity of the fluid increases hence an increase in the interaction of the magnetic field and the fluid resulting in increased magnetic induction.

From Figure 5, the velocity decreases with the increase in the injection parameter. Injection results in the thickening of the boundary layer hence a decrease in the velocity profiles.
Injection increases the pressure in the fluid and with constant discharge, the velocity decreases.

From Figure 6, the temperature decreases with the increase in the injection parameter. Injection leads to reduced velocities, the conversion of kinetic energy into thermal energy is also reduced hence a reduction in the temperature of the fluid.

From Figure 7, magnetic induction decreases with the increase in the injection parameter. As the injection parameter is increased, the velocity of the fluid decreases hence a decrease in the rate of interaction between the magnetic fluid and the inclined magnetic field which leads to reduced magnetic induction

From Figure 8, the magnetic induction increases with the increase in the Hartmann number. The increase in the Hartmann leads to the decrease in the viscous forces hence increase in the velocities fluid molecules thus increased magnetic induction.

From Figure 9, the temperature increases with the increase in the Hartmann number. As the Hartmann number increases, the effect of the viscous drag reduces and since viscosity and temperature and inversely, related, the temperature increases.

From Figure 10, the velocity of the fluid increases with the increase in the Hartmann number. As the Hartmann number increases, the effect of the viscous drug that opposes motion reduces hence increase in the velocities.

From Figure 11, the magnetic induction increases with the increases in the Joules heating parameter. As heating parameter increases the amount of current through the fluid increases since from the joules first law, the power of heating generated by an electric conductor is proportional to the product of its resistance and the square of its current. With the increase in the electric current applied, there is an increase in magnetic induction.

From Figure 12, the velocity decreases with increase in the unsteadiness parameter. The unsteadiness parameter increases, there is the thickening of the boundary layer due to no-slip condition and viscous drag which results in reduced velocities.

From Figure 13, the Magnetic induction reduces with the increase in the unsteadiness parameter. As the unsteadiness parameter increases, the velocity of the fluid is reduced due to the thickening of the boundary layer and the effect of the viscous drag hence reduced magnetic induction.

From Figure 14, the velocity increases with the decrease in the wedge angle. Using the different values of the wedge angle parameter $m$, i.e.

$$
\begin{aligned}
& m=\frac{1}{2}=0.5, \alpha=\frac{\pi}{3}=60^{\circ}, m=\frac{1}{3}=0.33 \ldots, \alpha=\frac{\pi}{4}=45^{0}, \\
& m=\frac{1}{4}=0.25, \alpha=\frac{\pi}{5}=36^{\circ}, m=\frac{1}{5}=0.2, \alpha=\frac{\pi}{6}=30^{\circ}
\end{aligned}
$$

As the wedge angle $\alpha$ decreases, the area of the wedge decreases and with a constant discharge $Q, Q=A V$, the velocity increases. As the area decreases, pressure increases hence an increase in the force in the fluid resulting in 
increased velocity.

From Figure 15, as the wedge angle $\alpha$ decreases, the magnetic induction increases. As the wedge angle $\alpha$ decreases, the area of the wedge decreases and with constant discharge, the velocity of the fluid increases and since magnetic induction and the velocity of the fluid increases and since magnetic induction and velocity of the fluid is directly proportional, the magnetic induction increases due to the increase in velocity.

\section{Conclusion}

The unsteady Jeffrey-Hamel flow in the presence of the inclined magnetic field with suction and injection has been investigated and the effect of various parameters discussed. The velocity and the magnetic induction profiles start at a maximum which is at the centerline velocity and decrease to zero which is the wall velocity due to the no-slip condition. The effect of varying various fluid flow variables is more pronounced between the center line and the walls of the divergent channel. Increase in the suction parameter leads to an increase in the velocity, temperature, and magnetic induction profiles while an increase in the injection parameter results in a decrease of the velocity, temperature, and magnetic induction profiles. The temperature and the magnetic induction profiles increase with the increase in the Hartmann number and the joule heating parameter while the velocity and the magnetic induction profiles decrease with the increase in time through the increase in the unsteadiness parameter due to the thickening of the boundary layer. The wedge angle plays a significant role since its decrease leads to reduced velocities and reduced magnetic induction as the effect of the boundary layer becomes more pronounced in the flow region.

\section{Nomenclature}

$\begin{array}{ll}\rho & \text { The density of the fluid } \\ r & \text { The radius of the channel } \\ \theta & \text { The angle of the channel } \\ \alpha & \begin{array}{l}\text { The angle from the centerline to the wall of the } \\ \text { channel }\end{array} \\ H & \text { Inclined Magnetic field } \\ u_{r}, u_{\theta} & \text { Velocity along } r, \theta \text { direction } \\ \beta & \text { The angle of inclination of the magnetic field } \\ T & \text { Temperature } \\ t & \text { Time } \\ \Phi & \text { Viscous dissipation function } \\ \mathbf{H} & \text { Induced Magnetic field } \\ \mathbf{B} & \text { Total magnetic field } \\ \mu & \text { Dynamic viscosity } \\ K & \text { Thermal diffusivity } \\ \sigma & \text { Electrical conductivity } \\ P r & \text { Prandtl number } \\ \operatorname{Re} & \text { Reynold number } \\ R_{m} & \text { Reynold magnetic number }\end{array}$
Ec Eckert number
$J \quad$ Joule heating parameter
$\mathrm{Ha}$ Hartmann number
$G_{r(C)} \quad$ Grashof Concentration number
$G_{r(T)} \quad$ Grashof Temperature number

\section{References}

[1] Jeffery, G. B. "L. The two-dimensional steady motion of a viscous fluid." The London, Edinburgh, and Dublin Philosophical Magazine and Journal of Science 29, no. 172 (1915): 455-465.

[2] Hamel, Georg. "Spiralförmige Bewegungen zäher Flüssigkeiten." Jahresbericht der Deutschen mathematikerVereinigung 25 (1917): 34-60.

[3] W. I. Axford, "The magnetohydrodynamic Jeffrey-Hamel problem for a weakly conducting fluid," The Quarterly Journal of Mechanics and Applied Mathematics, vol. 14, pp. 335-351, 1961.

[4] G. Domairry and A. Aziz, "Approximate analysis of MHD squeeze flow between two parallel disks with suction or injection by a homotopy perturbation method," Mathematical Problems in Engineering, vol. 2009, Article ID 603916, 19 pages, 2009.

[5] Imani, A. A., Rostamian, Y., Ganji, D. D., \& Rokni, H. B. (2012). Analytical investigation of Jeffery-Hamel flows with high magnetic field and nanoparticle by rvim.

[6] Asadullah, M., Khan, U., Manzoor, R., Ahmed, N., \& MohyudDin, S. T. (2013). MHD flow of a Jeffery fluid in converging and diverging channels. Int. J. Mod. Math. Sci, 6 (2), 92-106.

[7] Khan, U., Ahmed, N., Zaidi, Z. A., Jan, S. U., \& Mohyud-Din, S. T. (2013). On Jeffery-Hamel flows. Int J Mod Math Sci, 7 (3), 236-247.

[8] Sheikholeslami, M., Mollabasi, H., \& Ganji, D. D. (2015). Analytical investigation of MHD Jeffery-Hamel nanofluid flow in non-parallel walls. International Journal of Nanoscience and Nanotechnology, 11 (4), 241-248.

[9] Zubair Akbar, M., Ashraf, M., Farooq Iqbal, M., \& Ali, K. (2016). Heat and mass transfer analysis of unsteady MHD nanofluid flow through a channel with moving porous walls and medium. AIP Advances, 6 (4), 045222.

[10] Alam, M. S., Haque, M. M., \& Uddin, M. J. (2016). The convective flow of nanofluid along with a permeable stretching/shrinking wedge with second-order slip using Buongiorno's mathematical model. International Journal of Advanced in Applied Mathematics and Mechanics, 3 (3), 7991.

[11] Nagler, J. (2017). Jeffery-Hamel flow of non-Newtonian fluid with nonlinear viscosity and wall friction. Applied Mathematics and Mechanics, 38 (6), 815-830.

[12] Ochieng, F. O., Kinyanjui, M. N., \& Kimathi, M. E. (2018). Hydromagnetic Jeffery-Hamel Unsteady Flow of a Dissipative Non-Newtonian Fluid with Nonlinear Viscosity and Skin Friction. Global Journal of Pure and Applied Mathematics, 14 (8), 1101-1119. 
[13] Sattar, M. A. (2013). Derivation of the similarity equation of the 2-D unsteady boundary layer equations and the corresponding similarity conditions. American Journal of Fluid Dynamics, 3 (5), 135.

[14] Alam, M. S., \& Huda, M. N. (2013). A new approach for local similarity solutions of an unsteady hydromagnetic free convective heat transfer flow along a permeable flat surface.
International Journal of Advances in Applied Mathematics and Mechanics, 1 (2), 39-52.

[15] Alam, M. D. S., Khan, M. A. H., \& Alim, M. A. (2016). Magnetohydrodynamic Stability of Jeffery-Hamel Flow using Different Nanoparticles. Journal of Applied Fluid Mechanics, 9 (2). 